\title{
Principes de base de la conception des lasers à excimères pompés par décharge
}

\author{
M. Makarov
}

SOPRA, 26 rue Pierre Joigneaux, 92270 Bois-Colombes, France

\begin{abstract}
On parle d'application des lasers à excimères depuis vingt ans. Et depuis vingt ans on parle des problèmes de leur utilisation - ils sont fragiles, capricieux, peu fiables et chers. Mais pourquoi dans ce cas parle-t-on encore de ce type de laser? Tout simplement parce qu'aujourd'hui il n'existe pas d'autres moyens de générer un faisceau dense de photons énergétiques. Est-ce une situation sans issue?
\end{abstract}

\section{INTRODUCTION}

On peut distinguer trois périodes de l'histoire des lasers à excimères.

La première période, dite période d'EXPLORATION, dura approximativement de 1975 à 1985 . C'était la fièvre des excimères - chaque laseriste considérait de son devoir de faire ce laser à la mode. On pouvait trouver une gamme complète de caractéristiques énergétiques, temporelles, spectrales etc. [1]. La plupart de ces lasers ne représentaient que des appareils scientifiques de type « fait maison », mais les premières séries industrielles avaient déjà été lancées. La période d'TNDUSTRIALISATION commençait. Vers la fin des années 80 , le marché était définitivement établi et les lasers proposés se transformaient en vrais produits. On avait gagné en qualité. En revanche, après l'arrêt des programmes de recherche, on avait perdu en richesse de choix - les fabricants de lasers ayant choisi, bien évidemment, les systèmes les plus simples à réaliser. Et une fois ce choix fait, ils ne le changent plus. Leurs catalogues ne proposent que des variations (plus ou moins intéressantes) sur un thème donné - quelques centaines de millijoules dans une impulsion de quelques dizaines de nanosecondes. Les exceptions sont rares. Voilà pourquoi nous avons aujourd'hui une situation tout à fait paradoxale. Ce n'est pas le produit qui est adapté aux demandes, mais ce sont des applications qui s'adaptent aux propositions du marché existant. Comme conséquence, les nouvelles technologies se trouvent souvent bloquées dès le départ parce qu'elles ne peuvent pas concurrencer les technologies classiques qui sont déjà bien optimisées. Pour résoudre ce problème il faut inverser la situation et trouver une façon de fabriquer des lasers «sur mesure». C'est une période d'ADAPTATION à laquelle nous sommes confrontés aujourd'hui.

\section{ASPECT GENERAL DU PROBLEME}

Du point de vue technique, fabriquer sur mesure c'est avoir un produit facilement adaptable aux demandes particulières, c'est-à-dire un produit de base qui peut être légèrement modifié pour avoir les caractéristiques demandées (on parle ici, bien entendu, d'une certaine gamme limitée de caractéristiques possibles). Du point de vue physique, le problème est de garder dans chaque mode opérationnel les conditions optimales de fonctionnement du système. Plus ce système est complexe, plus l'optimisation globale est difficile. Théoriquement tous les sous-systèmes peuvent être optimisés, mais leurs optima ne doivent pas impérativement cö̈ncider. On ne peut donc parler que d'optimisation approximative quand chaque élément est proche de son fonctionnement optimal et que l'ensemble entier est optimal dans la mesure du possible. Pour ce faire, il faut d'abord révéler les éléments essentiels du système, puis établir pour chacun sa zone opérationnelle favorable et enfin trouver, si c'est possible, l'intersection de ces zones. 


\section{LASER A EXCIMERES COMME UN SYSTEME COMPEX}

Dans le cas particulier des lasers à excimères pompés par décharge, on peut distinguer trois soussystèmes principaux - le générateur d'impulsions électriques, le mélange gazeux à haute pression et le résonateur optique. Normalement, l'optimisation optique ne pose guère de problèmes. La formation de l'avalanche photonique est un processus final qui n'a pas d'influence rétroactive sur les processus antérieurs d'excitation du milieu actif. Par contre, l'optimisation du circuit électrique impulsionnel amorti par une décharge instable est beaucoup plus compliquée. La décharge est un système physique auto-organisé, qui s'adapte aux conditions externes imposées par le circuit électrique. En revanche, cette charge non linéaire a une influence forte sur le fonctionnement du générateur. En effet, quand on fait varier les conditions initiales, on ne change pas seulement le comportement du plasma, mais aussi, en conséquence, les caractéristiques du pulseur. C'est pour cela que l'optimisation d'un laser à excimères par rapport à un certain paramètre n'a aucun sens physique.

\section{ALGORITHME D'OPTIMISATION D'UN LASER A EXCIMERES}

Le rôle du circuit électrique est de délivrer au plasma actif une certaine quantité d'énergie. Le rôle de la décharge est de l'accepter, dans la limite de sa capacité, et de la transformer en une autre qualité. Ce flux énergétique doit être équilibré.

Quelle que soit la tension appliquée (au-dessus du seuil de déclenchement), le plasma tend vers un état stationnaire [1]. Dans cet état, la multiplication électronique est équilibrée par l'attachement aux molécules électronégatives de l'halogène. Le champ électrique local du plasma se stabilise. Sa valeur stationnaire ne dépend que de la composition du mélange gazeux. Le transfert énergétique du générateur au plasma est optimal quand la tension initiale appliquée est le double de la tension stationnaire (critère $A$ ).

En ce qui concerne les lasers, on a besoin d'un milieu actif homogène. En réalité, la décharge à excimères est instable et sa structure spatiale a tendance à se dégrader. Il y a deux raisons fondamentales à ce phénomène : la capacité limitée de l'émission électronique des électrodes et l'épuisement local de la réserve de l'halogène. On ne peut donc pas éviter cette dégradation, mais on peut la ralentir. Pour cela, il faut que le courant de la décharge dépasse rapidement (critère $B$ ) une limite inférieure (critère $C$ ).

Du point de vue de la transformation de l'énergie, la consommation de l'halogène est un processus positif. En effet, chaque acte d'émission stimulée signifie la destruction de quelques molécules halogènes. Comme leur réserve est limitée, l'énergie photonique d'extraction et l'énergie électrique consommable sont également limitées (critères $D$ et $E$ ).

Ces cinq critères établissent bien la zone opérationnelle optimale du laser [2]. Les valeurs approximatives du champ électrique stationnaire, de la densité du courant crête, de la durée d'impulsion, des énergies spécifiques d'extraction et d'excitation sont connues d'après les expériences et les calculs. Ils nous permettent d'estimer les paramètres du système entier. Quelles que soient les caractéristiques du laser demandées, on peut trouver la géométrie nécessaire du volume actif, la configuration du circuit électrique et les conditions initiales optimales.

\section{PETIT EXEMPLE}

Comme exemple de cette «fabrication sur mesure», considérons les lasers à excimères que SOPRA a développé dans le cadre de différents projets. Ces lasers couvrent une gamme complète de caractéristiques énergétiques et temporelles de $1 \mathrm{~mJ} / 1 \mathrm{~ns}$ à $60 \mathrm{~J} / 200 \mathrm{~ns}$. Bien évidemment, une production aussi flexible ne fut possible que grâce au savoir-faire de l'optimisation préliminaire.

\section{Références}

1. R. S. Taylor, Appl. Phys. B 41, 1-24 (1986)

2. M. Makarov, Rev. Sci. Instrum. 68, 3975-3988 (1997) 